\title{
II. Zweierlei Vergangenheiten
}

Eine komparatistische Analyse der Bewältigungsprozesse hat zunächst von den wesentlichen Spezifika der zugrundeliegenden Vergangenheiten auszugehen und sich neben den Unterschieden der politischen Systeme auch die Dimensionen der Verbrechen zu vergegenwärtigen, um die es dabei ging bzw. geht. Die prinzipielle Möglichkeit eines Vergleichs deutscher und japanischer "Vergangenheitsbewältigung" wird im Blick auf die unvorstellbare Zahl an Todesopfern, die auch der vom japanischen Kaiserreich entfesselte "Großostasiatische Krieg"1 zwischen 1931/37 und 1945 gefordert hat ${ }^{2}$, unmittelbar deutlich 3 . Von den etwa 20 Millionen Menschen, die infolge „normaler" militärischer Kampfhandlungen sowie aufgrund von Verbrechen unterschiedlichster Art starben, entfielen nach amtlichen Schätzungen allein 9,3 Millionen auf China, das darüber hinaus 9,5 Millionen Verwundete und 2,9 Millionen von der japanischen Armee verschleppte und später vermißte Menschen beklagte. Das Flüchtlingsproblem hatte ein gigantisches Ausmaß. Schätzungsweise 50 bis an die 100 Millionen flohen vor den Kampfhandlungen. Von den japanischen Truppen umgebracht wurden wahrscheinlich 3,9 Millionen chinesische Zivilisten und Kriegsgefangene. Schon dic Verluste unter letzteren betrugen zwischen 500000 und einer Million Menschen ${ }^{4}$.

Das später als „Dreistrahlentaktik“ aus Töten, Plündern und Niederbrennen bezeichnete brutale Vorgehen des japanischen Militärs in China gipfelte an der Jahreswende 1937/38 nach der Einnahme Nankings in einem Massa-

1 So der euphemistische, vom japanischen Regime selbst verwendete Begriff, der allerdings gegenüber dem „Pazifischen“ oder "Japanisch-Chinesischen“ Krieg zumindest den Vorteil hat, daß er sowohl die maritimen wie die festländischen Kriegsschauplätze umfaßt. Nüchtern und präzise läßt sich am besten vom „Ostasiatisch-Pazifischen Krieg“ sprechen.

2 In Ostasien läßt sich der Beginn des Zweiten Weltkriegs auf den - in Japan so genannten „China-Zwischenfall“ an der Marco-Polo-Brücke vom 7. Juli 1937 datieren. Vgl. Kuhn, Der Zweite Weltkrieg in China, S. 17. Allerdings hatte der chinesisch-japanische Krieg bereits mit dem „Mandschurischen Zwischenfall“ im September 1931 und der folgenden Besetzung von Mukden und der gesamten Mandschurei begonnen.

3 Zur Opferbilanz des Zweiten Weltkriegs im Fernen Osten vgl. Fuhrt, Erzwungene Reue, S. 20-24. Vertiefend Tanaka, Hidden Horrors; Li, The Scarch for Justice; Rees, Horror in the East; und nach wic vor die klassische amerikanische Studie von Russel, The Knights of Bushido.

4 Dabei ist in völkerrechtlicher Hinsicht allerdings zu berücksichtigen, daß dem Zweiten Chinesisch-Japanischen Krieg überhaupt keine Kriegserklärung zugrunde lag und daß Japan das - die Behandlung von Kriegsgefangenen regelnde - Genfer Protokoll vom 27. Juli 1929 nicht ratifiziert hatte. Erst nach Pearl Harbor gab Tokio seine Zustimmung zum Genfer Protokoll. Vgl. Kuhn, Der Zweite Weltkrieg in China, S. 30. 
ker, dem nach mehr als vorsichtigen Schätzungen 40000 Menschen, nach neueren Forschungen bis zu 200000 Einwohner der Stadt zum Opfer fielen ${ }^{5}$. Beim Versuch, Nordchina von jedem Einfluß des kommunistischen Südens abzuschotten und die Bevölkerung zu diesem Zweck in eine sogenannte Kollektivsiedlung $\mathrm{zu}$ treiben, wurden alle antijapanischen Kräfte ausnahmslos erschossen, Lebensmittel und Brennstoffe geraubt. Einwohnerschaften ganzer Dörfer wurden „routinemäßig ... massakriert“6, wenn sie im Verdacht standen, die Guerilla oder die chinesische Armee zu unterstützen. Allein im Rahmen dieser Strategie der „Ausweitung des Niemandslands" in Nordchina in den Jahren 1941 bis 1945 töteten japanische Soldaten über 2 Millionen Chinesen”. Daß Nanking kein „Betriebsunfall“ war ${ }^{8}$, erhellen neben vielen kleineren Greueltaten vor allem auch die Massaker bei der Eroberung Singapurs im Februar 1942 oder die Zerstörung Manilas Ende 1944, die Zehntausende Opfer kostete und von Massenvergewaltigungen, Folter und Verstümmelungen begleitet war?.

Hinzu kamen die Menschenversuche der berühmt-berüchtigten Einheit 731 in der Mandschurei, die der Herstellung bakteriologischer Waffen dienten, welche 1939/40 auch zum Einsatz gelangten ${ }^{10}$, sowie eine ganze Reihe weiterer - kaum bekannter - medizinischer Experimente durch japanische Ärzte an vielen anderen Orten ${ }^{11}$. Ein in letzter Zeit auch in der westlichen Öffentlichkeit stärker beachtetes düsteres Kapitel der japanischen Militärgeschichte schrieb die Zwangsrekrutierung von 50000 bis 200000 „Trostfrauen“, meist junge Mädchen vor allem aus Korea, China und anderen eroberten südostasiatischen Ländern, die sich japanischen Soldaten prostituieren mußten, unter menschenunwürdigen Verhältnissen in Militärbordellen hausten und am Kriegsende oft genug kaltblütig umgebracht wurden ${ }^{12}$. Besonders zu erwähnen sind zudem die 500000 Koreaner und Taiwan-Chinesen, die als zwangsrekrutierte Soldaten der japanischen Armee fielen, sowie die 40000 koreanischen Zwangsarbeiter, die bei den Atombombenabwürfen über Hiroshima und Nagasaki ums Leben kamen. Die Gesamtzahl der zur Schwerstarbeit gezwungenen Koreaner belief sich bis Kriegsende auf etwa 2 Millionen, wovon Tausende durch Unfälle, Mord oder Selbstmord starben.

5 Vgl. vor allem auch Honda, The Nanjing Massacre. Honda geht allerdings von „nur" etwas über 100000 Opfern aus. Ebd., S. XIII; eine knappe Zusammenfassung der Ereignisse und ihrer Rezeption in der japanischen Öffentlichkeit bietet Ishida, Das Massaker von Nanking.

6 Cohen, Öffentliche Erinnerung, S. 62.

7 Vgl. Ishida, Der „totale Krieg“, S. 443.

8 Dies zu suggerieren, wirft David Cohen Iris Chang vor. Cohen, Öffentliche Erinnerung, S. 62.

9 Ebd., S. 63. Zu den Ereignissen in Singapur Thürk, Singapore, vor allem S. $151 \mathrm{ff}$, und Warren, Singapore 1942. Zur japanischen Politik auf den Philippinen Herde, Großostasiatische Wohlstandssphäre.

10 Williams/Wallace, Unit 731.

11 Cohen, Öffentliche Erinnerung, S. 57.

12 Hierzu das Standardwerk von Yoshimi, Comfort Women. 
Aber auch die Todesrate unter den über 130000 alliierten Soldaten in japanischer Kriegsgefangenschaft war mit 27,1\% (35756 Menschen) extrem hoch; sie übertraf die Quote der in deutschen und italienischen Kriegsgefangenenlagern umgekommenen westalliierten Soldaten (4\%) um das Siebenfache ${ }^{13}$ und erinnert sehr an die Grausamkeit, mit der die sowjetischen Kriegsgefangenen vom Dritten Reich behandelt wurden. Allein beim Bau der berüchtigten Burma-Bahn, den Mitte der 1950er Jahre der HollywoodFilm „Die Brücke am Kwai“ in Erinnerung rief, sind vermutlich weit mehr als 10000 von 60000 eingesetzten alliierten Kriegsgefangenen umgekommen. Sie teilten das Schicksal mit an die 270000 Zwangsarbeitern aus China, Malaysia, Birma oder Java, von denen etwa 50000 elend im Dschungel zugrunde gingen ${ }^{14}$.

Ist es vor dem Hintergrund derartiger Verbrechen angemessen zu sagen, die Brutalität, mit der japanische Truppen häufig Kriegsgefangene wie Zivilbevölkerung behandelten, sei „weit entfernt von der Unmenschlichkeit“ gewesen, „mit der das Dritte Reich seine Völkermorde plante und verübte“ 15 ? Darf man sich ein „moralisches“ Urteil darüber crlauben, ob die Qualen der von japanischer Soldateska massenhaft vergewaltigten und schließlich mit in die Vagina gestoßenen Bambusrohren zurückgelassenen chinesischen Frauen oder vieler anderer ähnlich grausam zu Tode gekommener Opfer des Krieges im fernen Osten „weit entfernt" waren von dem jedes menschliche Fassungsvermögen übersteigenden Leiden der europäischen Juden und anderer Verfolgter im Herrschaftsbereich des Nationalsozialismus? Fragen gegen eine derartige Form des Aufrechnens stellen sich, auch wenn die Bilanzen der NS-Verbrechensstatistik die des japanischen Ultranationalismus übersteigen.

Nicht nur fielen dem von Hitler entfesselten Krieg auf den europäischen und nordafrikanischen Schlachtfeldern, vor allem aber in Rußland, noch mehr Soldaten und Zivilisten zum Opfer als dem ostasiatisch-pazifischen Orlog ${ }^{16}$. Auch der systematische Massenmord erreichte im Dritten Reich eine noch größere Dimension. Hat Werner Gruhl die Zahl der „deaths from genocide, barbarity, forced labor, and caught in combat“ für das militaristische Japan auf ca. 7 Millionen berechnet ${ }^{17}$, so wird die Zahl der unter dem Nationalsozialismus Ermordeten nach derzeitigem Kenntnisstand auf zwischen 12 und 14 Millionen geschätzt: allein 5,6 bis 5,7 Millionen Menschen jüdischer Herkunft, mindestens 100000 Sinti und Roma, knapp drei Millio-

13 Tanaka, Hidden Horrors, S. 2 f.

14 Ebd., S. 120; vertiefend McCormack/Nelson, The Burma-Thailand Railway, sowie das jüngst erschienene Buch von Kratoska, Thailand-Burma railways.

15 Haasch, Japan, S. 44.

16 Die schwankenden Zahlenangaben sind schwer kommensurabel zu machen. Vgl. aber die sorgfältigen statistischen Berechnungen von Gruhl, The Great Asian-Pacific Crescent of Pain, S. 256.

17 Ebd. 
nen in Kriegsgefangenschaft geratene Staatsbürger der Sowjetunion und jeweils weit über eine Million Zivilisten in Polen und in den besetzten Gebieten der UdSSR sowie mehrere Hunderttausend in Jugoslawien, davon eine große Zahl Opfer von Massenerschießungen, selektiver Hungerpolitik und sogenannter „Bandenkampfaktionen“ gegen Partisanen ${ }^{18}$. Hinzu kamen Morde an Behinderten und Kranken, Verbrechen bei der Bekämpfung des Widerstandes im eigenen Land, aber auch das lange kaum erforschte Kapitel der Zwangsprostitution zehntausender Frauen in den KZ-Bordellen der SS und in Wehrmachtsbordellen, von denen viele an Geschlechtskrankheiten starben oder umgebracht wurden ${ }^{19}$. Wer sich Art und Umfang dieser Massenverbrechen vergegenwärtigt, an denen zu einem nicht unerheblichen Teil auch Angehörige der Wehrmacht beteiligt waren ${ }^{20}$, wird schwerlich Robert Leckies - auf das Massaker von Nanking bezogene - Ansicht teilen, wonach keine der Greueltaten, „die die Nazis unter Hitler auf dem Weg zu ihren Siegen begingen, ... sich mit denen der japanischen Soldaten unter General Matsui Iwane messen" konnte ${ }^{21}$.

Gehörte zur gemeinsamen Motivstruktur der nationalsozialistischen Täter das Denken in rassistischen Kategorien, so spielten auch bei den japanischen Verbrechen rassistische Affekte eine wichtige Rolle. Die Rassenlehre des Professors Okawa Shumei von der Assimilierung der fünf asiatischen Rassen und dem irdischen Paradies, das die rassisch überlegenen Japaner zu errichten hätten, hat schon in den 1930 er Jahren die politischen Aktionen der Militärs stark beeinflußt ${ }^{22}$. Auch in der übrigen Gesellschaft war die selbst von Schlagersängern popularisierte Vorstellung weit verbreitet, einem direkt von den Göttern abstammenden Herrenvolk anzugehören, dessen Aufgabe es sei, die asiatischen Völker und überhaupt alle farbigen Rassen von der Schmach der Fremdherrschaft zu befreien. Die traditionelle Gegnerschaft gegen den europäischen Kolonialismus wuchs sich dabei zum Haß gegen die weiße Rasse und vor allem gegen die Anglo-Amerikaner („teuflische Bestien") aus. Aber auch die Verachtung gegenüber anderen, als minderwertig erachteten asiatischen Völkern, besonders Koreaner und Chinesen, nahm zu${ }^{23}$. So wie weiße Kriegsgefangene wurden auch die Chinesen wie ein japanischer Veteran in einem Fernsehinterview später einräumte -

18 Vgl. D. Pohl, Verfolgung und Massenmord, S. $153 \mathrm{f}$.

19 Paul, Zwangsprostitution, S. 135.

20 Christian Hartmann hat in einem vielbeachteten Aufsatz jüngst eine vorläufige Bilanz des Forschungsstandes gezogen und deutlich gemacht, daß, falls die geschätzte "Täterquote“ von fünf Prozent zuträfe, eine halbe Million deutscher Soldaten "gegen Recht und Sitte verstoßen hätten". Christian Hartmann, Verbrecherischer Krieg - verbrecherische Wehrmacht?, S. 71.

21 Leckie, Delivered from Evil, S. 303.

22 Vgl. hicrzu Shigemitsu, Die Schicksalsjahre Japans, S. 33, 67, 95.

23 Nakai, Die Entmilitarisierung, S. 8 f. 
gar nicht als Menschen betrachtet; und sie konnten deshalb ohne Bedenken ermordet werden ${ }^{24}$.

Die japanische Offiziersschule hatte schon 1933 ein Lehrbuch herausgegeben, das die Tötung chinesischer Gefangener im Hinblick darauf rechtfertigte, daß sich darunter eine Unmenge standesamtlich nicht Registrierter und Obdachloser befinde. In den von der japanischen Besatzungsmacht in der Mandschurei zusammengestellten „Grundideen“ hieß es 1936 programmatisch, es gehe im Sinne einer weltweiten Tennô-Herrschaft darum, „die Mangelhaften anderer Völker zu ergänzen, die Faulen zu peitschen und die Ungehorsamen gehorchen zu lassen "25. Den japanischen Kommandeuren in China wurde am 5. August 1937 vom Tokioter Heeresministerium ausdrücklich der Befehl erteilt, bei der Behandlung der Kriegsgefangenen jede völkerrechtliche Bestimmung außer acht zu lassen. Ohnehin sahen viele japanische Soldaten in der Ermordung von Chinesen gleichsam ein religiöses Verdienst im Rahmen eines "heiligen“, vom Tennô geführten Krieges: „Sie waren überzeugt davon, daß keiner der Feinde des Kaisers im Recht sein konnte, je brutaler sie also ihre Gefangenen mißhandelten, desto deutlicher zeigten sie ihre Kaisertreue. "26 Hinzu kam indes, daß die japanischen Soldaten von ihren Vorgesetzten oft aufs brutalste schikaniert wurden und das sich dabei aufstauende riesige Gewaltpotential sozusagen an ihren chinesischen Opfern abbauten ${ }^{27}$.

Der im Militär verwurzelte Rassismus war bereits im Erziehungssystem Japans angelegt ${ }^{28}$. Als Antwort auf die erzwungene Öffnung des Landes durch die schwarzen Schiffe des amerikanischen Commodore Matthew C. Perry 1853 wurden im Zuge der Meiji-Reform Ende des 19. Jahrhunderts Orientierungsmuster für das Bildungswesen entwickelt, die in einem bis 1945 geltenden kaiserlichen Erziehungsedikt von 1890 kulminierten. Das in allen Schulen täglich vor Unterrichtsbeginn den - sich ehrfürchtig Richtung Kaiserpalast verneigenden - Schülern verlesene quasireligiöse Dokument stellte in seiner Mischung nationalshintôistischer und konfuzianischer Elemente gleichsam „die Inkarnation und Materialisation der geistigen Essenz Japans“ dar ${ }^{29}$. Ideologische Richtschnur wurde fortan das sogenannte kokutai-Denken, die Überzeugung vom unverwechselbaren und besonderen Charakter der japanischen Nation, einer göttlichen Familie mit dem Tennô als Oberhaupt, den die Untertanen - einer strengen Hierarchie gehorchend - wie einen Vater in Loyalität und Kindesliebe verehrten.

24 Buruma, Erbschaft der Schuld, S. 221.

${ }^{25}$ Ishida, Der „totale Krieg“, S. 440 (Zitat), 443.

26 So berichtet ein amerikanischer Priester, der im Sugamo-Gefängnis inhaftierte ehemalige japanische Lagerkommandanten danach fragte, warum sie Kriegsgefangene so mißhandelt hätten. Buruma, Erbschaft der Schuld, S. 221.

27 So die Argumentation von Tanaka, Hidden Horrors.

28 Zum folgenden vgl. Rosenzweig, Erziehung, S. 50-63.

29 Antoni, Kokutai - Das „Nationalwesen“, S. 45. 
Auf der Basis dieser jahrzehntelang vermittelten Nationalmoral ging die Erziehungspolitik während der japanischen Expansion dann auf eindeutig nationalistisch-militaristischen Kurs. Sie stellte den Wert der nationalen Tradition gegenüber dem westlichen Individualismus heraus und machte aus den Grundschulen „Nationale Schulen“, die neben Leibesübungen und Mathematik vor allem „nationale Studien“ betrieben. Den Schülern und Studenten, so hieß es in Unterrichtsdirektiven vom März 1943, „soll der kaiserliche Auftrag in Fernost und auf der ganzen Welt und die Lebensnotwendigkeit der nationalen Verteidigung bewußt gemacht werden". 30 Glaubhaft wirken vor diesem Hintergrund auch Episoden, wie sie der Historiker Ienaga Saburô schildert; daß etwa ein japanischer Lehrer in den 1930er Jahren einem Schüler, der sich scheute, einen Frosch zu sezieren, eine Kopfnuß gegeben und gesagt habe: „Warum weinst Du über einen mickrigen Frosch, wenn du groß bist, wirst du ein- oder zweihundert Gelbe umlegen müssen"31. In Anbetracht der Entwicklungen im Erziehungswesen und im Militär wird jedenfalls nachvollziehbar, weshalb Buruma die Ideologie, die der Barbarei der japanischen Truppen zugrunde lag, als „genauso rassistisch“32 bewertet hat wie Hitlers Nationalsozialismus mit seiner Vergötterung des arischen Herrenmenschen und seiner Verachtung für jüdische und slawische „Untermenschen“. Auch wem dieses Urteil zu apodiktisch scheint ${ }^{33}$, kommt doch nicht umhin, zumindest frappierende ideologische Parallelen zu konstatieren.

Eine andere Frage ist, wie systematisch sich die rassistische Weltanschauung in kriminelles Handeln umsetzte. Arnold Brackmann, der als junger Journalist dem Internationalen Militärtribunal für den Fernen Osten beiwohnte, kommt zu dem Schluß, daß vor allem das Massaker von Nanking „kein isoliertes Ereignis war, wie es in allen Kriegen vorkommt“, sondern von der Führung in Tokio ganz bewußt inszeniert wurde ${ }^{34}$. Auch Iris Chang berichtet vom Tagebuch eines japanischen Obersten, in dem es heißt: "Ich habe Order von meinem Vorgesetzten, alle Menschen hier umzubringen“35. Der an der Chuo-Universität Tokio lehrende vergleichende Genozidforscher Uwe Makino hat die Vergewaltigung von Nanking demnach in die Geschichte der Terrorgenozide eingeordnet, die nicht nur eroberungs-

30 Zit. nach Rosenzweig, Erziehung, S. 62.

31 Buruma, Erbschaft der Schuld, S. 220. Zum japanischen Rassismus, der sich stärker noch als aus der Herabsetzung der anderen Völker aus der Überhöhung der eigenen Rasse speiste, vgl. Dower, War without Mercy, S. $203 \mathrm{ff}$. Zum Rassismus als wechselseitiges Problem der japanisch-amerikanischen Bezichungen: Koshiro, Trans-Pacific Racisms.

32 Buruma, Erbschaft der Schuld, S. 220.

33 Der nationalsozialistische Antisemitismus erschöpfte sich schließlich nicht in Rassismus, sondern erfuhr seine Radikalität durch die Verknüpfung von biologistischen Vorstellungen und antisemitischer Mythologie.

34 Brackmann, zit. nach Chang, Die Vergewaltigung von Nanking, S. 184.

35 Chang, Die Vergewaltigung von Nanking, S. 223. 
strategische Ziele verfolgten, sondern von herrschenden Minderheiten auch als „Verwaltungstechnik“ zur Abschreckung echter oder potentieller Gegner eingesetzt wurden: „[...] der Terror hatte Funktion mit Blick auf die weiteren Ziele in China und Südostasien"36. Allerdings hat Ishida Yuji mit gutem Grund eingewandt, daß Nanking gerade nicht Teil der „Dreistrahlen-Taktik“ Japans, sondern „eher durch eine durch den Kriegsverlauf bedingte ,Spontaneität" gekennzeichnet“ war. Dieses bekannteste Massaker lasse sich nicht „durch einen Befehl des Generalstabs oder den Willen des Staates“ begründen, sondern „durch die Initiative der Kommandanten vor Ort und das Verhalten gewöhnlicher Japaner". Ansonsten aber unterstreicht auch Ishida die Planmäßigkeit der „genozidalen Übergriffe gegen die einheimische Bevölkerung" in Nordchina. ${ }^{37}$. In ähnlicher Weise hat Gavan McCormack die japanische Kriegführung als genozidal im Sinne der UNKonvention von 1948 bezeichnet: „acts committed with intent to destroy, in whole or in part, a national, ethnical, racial, or religious group" 38 . Klaus Marxen und Gerhard Werle, Rechtsprofessoren der Berliner HumboldtUniversität, gelangen ebenfalls zu der Einschätzung, die von Japan und Deutschland verübten schweren Menschenrechtsverletzungen im Zweiten Weltkrieg seien zwar „unterschiedlich nach Art und Umfang“ gewesen, aber doch „gleichermaßen systematisch“ 39 .

Auch wenn man demnach die These vertreten könnte, der nationalsozialistische Weltanschauungskrieg gegen die Sowjetunion erinnere „in seiner Maßlosigkeit an die Verbrechen der Japaner in China" 40 , so bleibt in der Gesamtbeurteilung beider Schreckensherrschaften doch ein wesentlicher rezeptionsgeschichtlich wirkmächtiger - Unterschied: Die übergroße Masse der Schandtaten des japanischen Ultranationalismus ging in Planung und Ausführung auf das Konto des Militärs und konnte - zutreffend oder nicht - jedenfalls relativ leicht als gleichsam herkömmliche, in allen Kriegen vorkommende Exzesse verharmlost werden, wie das im übrigen auch mit den Verbrechen der Wehrmacht in Deutschland nach 1945 teilweise geschah ${ }^{41}$, obwohl die Armee eben nicht nur Kriegsverbrechen im Sinne von Verstößen gegen das Kriegsvölkerrecht zu verantworten hatte, sondern darüber

36 Makino, Terror als Eroberungs- und Herrschaftstechnik, S. 349. Vgl. auch den eindrucksvollen Bericht eines deutschen Zeitzeugen: Wickert (Hg.), John Rabe: Der gute Deutsche von Nanking.

37 Ishida, Das Massaker von Nanking, S. 235.

38 McCormack, Reflections on Modern Japanese History, S. $266 \mathrm{f}$., $273 \mathrm{f}$.

39 Vgl. die Einführung der beiden Strafrechtler in dem von ihnen mitherausgegebenen Sammelband: Marxen u. a., Der Umgang mit Kriegs- und Besatzungsunrecht, S. 13,

40 Makino, Terror als Eroberungs- und Herrschaftstechnik, S. 352.

41 So mußte sich z. B. Hochkommissar John McCloy in bezug auf die Landsberger Häftlinge gegen Versuche wenden, „die jeweiligen Verbrechen als notwendige Kriegshandlungen abzutun“, und darauf verweisen, daß die Inhaftierten wegen der Teilnahme am NS-Rassenprogramm einsäßen. Brochhagen, Nach Nürnberg, S. 42 f. Vgl. auch Frei, Vergangenheitspolitik, S. 174 f., $188 f$ f. 
hinaus an spezifischen NS-Gewaltverbrechen beteiligt war, die mit den Kriegshandlungen im eigentlichen nichts zu tun hatten. In diesen Kontext gehört etwa auch der grausame, oft genug tödlich endende Mißbrauch von $\mathrm{KZ}$-Häftlingen durch skrupellose Militärärzte für die Forschung zu wehrwissenschaftlichen Zwecken ${ }^{42}$, in der man eine Analogie zu den Menschenversuchen der japanischen Einheit 731 sehen kann. Die Verbrechen der Wehrmacht machten aber - trotz ihrer erheblichen Größenordnung ${ }^{43}$ einen deutlich kleineren Anteil an den kriminellen Taten des Regimes insgesamt aus als dies für die Verbrechen des japanischen Militärs galt. Denn schließlich und vor allem war die nationalsozialistische Opferbilanz durch einen dieser Art noch nie vorgekommenen und teilweise industriell betriebenen Massenmord gekennzeichnet, der auf einem staatlichen Programm basierte, die europäischen Juden ,allein wegen ihrer Herkunft restlos und in kürzester Zeit zu ermorden" 44 , ohne daß sich an dieser Entscheidung auch nur nennenswerte interne Debatten im NS-Herrschaftsapparat entzündet hätten.

All dies mußte ebenso Folgen für den Charakter der späteren „Vergangenheitsbewältigung " in Deutschland und Japan zeitigen wie der Umstand, $\mathrm{da} ß$ sich die (politische) Verantwortung für das Unrechtsgeschehen in der Diktatur Hitlers zumindest viel klarer zuordnen ließ als im Staats- und Gesellschaftssystem des Tennô-Reiches. Dort hatte auf dem Höhepunkt der Weltwirtschaftskrise eben keine totalitäre Führer-Bewegung die Macht ergriffen und sich den Staat untertan gemacht, sondern hatte die Militärführung - mit Verständigungspolitik und Abrüstungsbereitschaft der TaishôDemokratie ${ }^{45}$ von jeher unzufrieden - den gescheiterten Putschversuch radikaler junger Offiziere vom 26. Februar 1936 dazu ausgenutzt, eine „legale, indirekte Herrschaft" ${ }^{" 46}$ des Militärs zu etablieren. Sie stützte sich zum einen auf das hohe Prestige der Truppe seit den siegreichen Kriegen gegen China (1894) und Rußland (1904/05), die zur Annexion Taiwans und Koreas geführt hatten ${ }^{47}$, zum anderen auf die verfassungsrechtlich normierte, von der Taishô-Demokratie angeblich untergrabene oberste Kommandogewalt des Tennô. Faktisch gewannen vor allem Stabsoffiziere des Heeres (im Range des Majors, Oberstleutnants oder Obersts) maßgeblichen politischen Einfluß; und zwar dadurch, daß sie die von einem nicht genehmen Premier vorgeschlagenen Armeeminister ablehnten, was regelmäßig zum Rücktritt des

42 Vgl. hierzu Mitscherlich/Mielke (Hg.), Medizin ohne Menschlichkeit.

43 Zwar hatten, mit Christian Hartmann zu reden, viele Soldaten wenig, aber doch eben auch „wenige [...] viel zu verantworten“. C. Hartmann, Verbrecherischer Krieg, S. 71.

44 D. Pohl, Verfolgung und Massenmord, S. 109.

45 Taishô bezeichnet die Jahre zwischen 1912 bis 1926, als unter dem 123. Tennô die demokratische Erneuerung des autoritären Kaiserregimes begann.

46 Kurozawa, Das System von 1940 und das Problem der politischen Führung in Japan, S. 144.

47 Vgl. Ishida, Die japanischen Kriegsverbrechen, S. 328. 
Regierungschefs selbst führte, dessen Rücktrittsersuchen gegenüber dem Tennô dann als „Opferung des Skeletts“ bezeichnet wurde ${ }^{48}$.

Den die Kabinette dominierenden Soldaten gelang es freilich nicht, die anderen Staatsorgane ganz unter ihre Kontrolle zu bringen. Vielmehr erwies sich das jetzt entstehende „System politischer Zusammenarbeit“ verschiedener Machtzentren in Militär und Staatsbürokratie im Verlaufe des Krieges als derart ineffizient, daß im Oktober 1940 mit der Gründung des Bundes zur Förderung der kaiserlichen Herrschaft der - abermals nicht richtig gelingende - Versuch unternommen wurde, eine neue politische Integrationsinstanz zu schaffen und die Führungskraft des Kabinetts zu stärken. Zur charismatischen Herrschaft eines Offiziers konnte es schon deshalb kaum kommen, weil nach der bis 1945 in Kraft bleibenden Meiji-Verfassung von 1889 der „heilige und unverletzliche“ Tennô das „Kaiserreich Groß-Japan beherrscht und regiert" (Artikel I und III) und auch als Oberbefehlshaber des Heeres und der Kriegsmarine fungiert hat ${ }^{49}$.

Trotz dieser theoretisch absoluten Autorität blieb der Kaiser aber „a transcendental and passive sovereign" 50 im Rahmen einer eigentümlichen Art von konstitutioneller Monarchie, die den rechtlichen Status des Kabinetts einfach offen ließ. So erfolgte die Ernennung zum Premierminister dergestalt, daß durch das „Organ“ des Naidaijin, d.h. des den Tennô beratenden „Persönlichen Palastministers“, ein Kandidat vorgeschlagen und dann per „Kaiserlichem Großbefehl“ ernannt wurde. Nur entbehrte die anerkannte Institution des Naidaijin, traditionell aus den Reihen der Jushin, der hohen Staatsbeamten, rekrutiert, einer rechtlichen Grundlage, sondern diente dazu, den Tennô im Falle etwaiger Kritik von jeder Verantwortung für die Ernennung abzuschirmen. Für das Kabinett als eigentlichem Zentrum der Exekutivgewalt gab es keinerlei verfassungsrechtliche oder auch nur gesetzliche Regelungen außer der Feststellung, daß es die konstitutionell normierte „Verwaltungsallmacht" des Tennô in dessen Vertretung und in alleiniger Verantwortung vor ihm ausübe. Das Kabinett war also „weder ein formelles Staatsorgan noch überhaupt eine Rechtsinstitution"51. Dennoch kann auf verfassungspraktischer Ebene argumentiert werden, die wirkliche Macht in Japan sei von im Namen des Tennô regierenden Ministern ausgeübt worden, die nach der Konstitution die Regierungsverantwortung trugen ${ }^{52}$. Und diese „nach westlichem Denken zwiespältige Stellung" habe zur Folge gehabt, daß der Kaiser nicht umhin gekommen sei, die „Entscheidungen der Regierungen vorbehaltlos gut(zu)heißen" ${ }^{\text {53 }}$.

48 Miyazawa, Rechtsprobleme der Kriegsverbrecherprozesse, S. 37.

49 Kurozawa, Das System von 1940, S. 147.

50 Hall, A Monarch for Modern Japan, S. 47.

51 Miyazawa, Rechtsprobleme der Kriegsverbrecherprozesse, S. 37.

52 Vgl. Wetzler, Kaiser Hirohito, S. 617.

53 Nakai, Die „Entmilitarisierung“, S. 19. 
Was die mangelnde juristische Normierung staatlicher Entscheidungsprozesse, ihr bloßes Gründen auf Herkommen, Überlieferung und Gewohnheit für die rechtliche und insbesondere strafrechtliche Verantwortung für Krieg und Kriegsverbrechen in Japan bedeutete, mag aus dem bemerkenswerten Streit darüber erhellen, ob der Kaiser selbst den Angriff auf Pearl Harbor befohlen habe oder ob er möglicherweise gar nichts davon wußte, weil seine Berater „ihm zu seinem Schutz diese Informationen absichtlich vorenthalten haben" 54 . Ähnlich charakteristisch sind die Aussagen führender Persönlichkeiten nach 1945 zur Kriegsschuld einer „Militärclique“, die laut Ex-Premier Shidehara Kijûro aus „jüngeren Armeeoffizieren“ bestand, deren Namen er aber nicht genau kenne, zumal das Kabinett ohnehin keine direkte Kontrolle über die Armee gehabt und kein Gesetz genau festgelegt habe, wer für die Aktionen des Militärs die Verantwortung trage. Auf die Frage, wo denn der Kopf der Armee gesessen habe, antwortete ein ehemaliger japanischer Wirtschaftsführer: „Nobody knows. It was like a current flowing through a large number of young officiers in the Army"55.

$\mathrm{Daß}$ schließlich selbst ein Armeeminister sich darauf berufen konnte, unter dem Druck seiner Untergebenen eine bestimmte Politik verfolgt zu haben oder gar „a manipulated robot" 56 gewesen zu sein, verweist auf das in tradierten japanischen Gesellschaftsstrukturen wurzelnde, auch nach 1936 funktionierende „System der Unverantwortlichkeiten“, wie es der Politikwissenschaftler Maruyama Masao beschrieben hat. Danach ist zwischen drei politischen Typen - dem tragbaren Schrein, den Beamten und den Gesetzlosen - zu unterscheiden. Der ranghöchste Schrein, das oberste Symbol der Autorität, wird von den Beamten wie ein Schrein an Feiertagen auf den Schultern getragen, bleibt aber gleichsam Ikone, während die tatsächliche Macht von den Beamten - Ministern, Verwaltungseliten, Generälen - ausgeübt wird. Auch diese wiederum werden indes von den niederen Rängen aus manipuliert, „von den Gesetzlosen, von Einzelgängern unter den Militärs, hitzköpfigen Offizieren draußen an der Front, von rasenden Nationalisten und anderen Agenten der Gewalt“57. Geschichte wird in diesem „System der Unverantwortlichkeiten“ zu einer endlosen Kette vollendeter Tatsachen, die wie Naturgewalten mysteriösen Ursprungs über die Menschen kommen. Gerät das System außer Kontrolle, wie dies in den 1930er Jahren der Fall war, „diktieren die gewalttätigen Gesetzlosen die Ereignisse, nervöse Beamte reagieren nur mehr darauf, und die Heiligkeit des (kaiserlichen, M.K.) Schreins rechtfertigt alles. "58 Was dies konkret hieß, war z. B. bei der

54 So Wetzler, Kaiser Hirohito, S. 628, unter Bezug auf Butow, Tojo and the coming of the War, S. $370 \mathrm{f}$.

55 Zit. nach Baerwald, The Purge of the Japanese Leaders, S. 15.

56 Ebd.

57 Buruma, Erbschaft, S. 218.

58 Ebd. 
Eroberung Nankings zu sehen, die General Matsui - unter Mißachtung von Befehlen der Regierung in Tokio und des Generalstabs - eigenmächtig begann. Erst als sich ein Erfolg der Operation abzeichnete, erteilte auch der Generalstab den Befehl zum Angriff auf die symbolträchtige Hauptstadt der chinesischen Republik ${ }^{59}$.

Die für den westlichen Beobachter einigermaßen rätselhaften Hierarchien im politischen und militärischen System Japans unterscheiden sich aber selbst dann noch fundamental von den Zuständen im Dritten Reich, wenn man dieses eher als polykratische Diktatur begreift. Neben der grundlegenden Differenz in der Ausprägung des Primats des Politischen war vor allem auch die Beziehung zwischen Regime und Volk in beiden Fällen offensichtlich ganz anders gelagert. Die nationalsozialistische Massenbewegung erschien später auch den amerikanischen Nachkriegsplanungsstäben mit dem - nicht in einer politischen Partei zu identifizierenden und unter keiner einheitlichen diktatorischen Führung stehenden - elitären japanischen Militarismus nicht vergleichbar ${ }^{60}$. Während dementsprechend die Potsdamer Beschlüsse der alliierten Siegermächte 1945 faktisch von einer kollektiven Verantwortung des deutschen Volkes für den Krieg ausgingen ${ }^{61}$, war in bezug auf das fernöstliche Inselreich von der „Macht und dem Einfluß jener“ die Rede, die „mit Täuschung und Irreführung das japanische Volk zum Wahn der Welteroberung verleitet“ hätten und „ein für allemal ausgeschaltet werden“ müßten 62 .

Ob diese Einschätzung der gesellschaftlichen Fundierung auch des japanischen Militarismus und Ultranationalismus tatsächlich ganz gerecht wurde, darf zumindest mit einem Fragezeichen versehen werden. Jedenfalls erzeugten die an Familie und Dorfgemeinschaft orientierten sozialen Verhaltensweisen in Japan eine viel größere gesellschaftliche Homogenität, als sie das Dritte Reich selbst durch Verführung und Gewalt jemals herzustellen vermochte. Ein mit der deutschen Opposition gegen Hitler vergleichbarer, bis zum Staatsstreich führender Widerstand gegen das Regime hat sich in Japan auch deshalb nicht entwickelt ${ }^{63}$. Zwar wurden dort zwischen 1928 und 1941 etwa 66000 Menschen wegen ihrer linken politischen Überzeugungen verhaftet, jedoch nur einmal (1944) aus politischen Gründen die

59 Ishida, Der „totale Krieg“, S. 441.

60 Rosenzweig, Erziehung, S. 108.

$61 \mathrm{Daß}$ die Rede von der Kollektivschuld „nicht allein cin Rezeptionsphänomen der Deutschen" war, zeigt auch ein näherer Blick auf dic angelsächsische Diskussion während des Krieges, in deren Verlauf „durchaus eine Anklage der gesamten deutschen Nation formuliert" wurde. Friedrich/Später, Britische und deutsche Kollektivschuld-Debatte, S. 89.

62 Ursachen und Folgen, Band 23, S. 493.

63 Zwar war die japanische Geschichte voll von bäuerlichen und soldatischen Aufständen, doch der Fehlschlag bewaffneter Revolten am Ende der Bürgerrechtsbewegung 1884 „wrote finis to armed struggle in Japan“. Ienaga, The Pacific War, S. 223; vgl. auch die weiterführenden Bemerkungen zum Thema „dissent and resistance“ in ebd., S. 203-223. 
Todesstrafe verhängt. Zudem hatten die Gefangenen die Möglichkeit, ihrer „falschen“ Einstellung abzuschwören (tenko), um aus der Haft entlassen zu werden ${ }^{64}$. Diese Widerrufe wurden zwar nicht direkt - mittels der im Dritten Reich üblichen Terrormaßnahmen - erpreßt, können aber doch als eine „subtile Form der Gewaltanwendung“ auf dem Wege spezifisch japanischer Sanktionsmechanismen der Gesellschaft verstanden werden ${ }^{65}$.

Gerade die traditionell homogene und gruppenorientierte Ordnung des japanischen Volkes war es aber auch, die von den Planern im amerikanischen Außenministerium als vorteilhaft für den angestrebten politischen Transformationsprozeß nach dem Krieg angesehen wurde. Gelänge es, die militaristisch-ultranationalistischen Eliten durch liberale Führungskräfte zu ersetzen, würde - so hoffte man - in der vertikal strukturierten japanischen Gesellschaft relativ leicht ein demokratischer Konsens von oben nach unten wachsen $^{66}$. Der Aufbau einer Demokratie schien den Japan-Experten im State Department allerdings nur unter der Schirmherrschaft des Tennô „als wichtigstem gesellschaftlichem Integrativ“ möglich, während sie für den Fall einer Beseitigung des traditionellen Kaisertums gewaltsame Proteste und Widerstand gegen die amerikanische Besatzungsmacht, bis hin zu einem Guerillakrieg, befürchteten ${ }^{67}$.

64 Mitchell, Thought Control in Prewar Japan, S. $191 \mathrm{ff}$.

65 Schieder, Kriegsregime des 20. Jahrhunderts, S. 35.

66 Rosenzweig, Erziehung, S. 108.

67 Ebd., S. 99 